\title{
Delivery of a chemotherapeutic drug using novel hollow carbon spheres for esophageal cancer
} \section{treatment}

Li Zhang, ${ }^{1, *}$ Mengchu Yao, ${ }^{2,3, *}$ Wei Yan, ${ }^{4, *}$ Xiaoning Liu, ${ }^{5}$ Baofei Jiang, ${ }^{6}$ Zhaoye Qian, ${ }^{2,3}$ Yong Gao, ${ }^{2,3}$ Xiao-jie Lu, ${ }^{7}$ Xiaofei Chen, ${ }^{2}$ Qi-long Wang $2,3,5$

'Department of Hematology, ${ }^{2}$ Department of Clinical Oncology, ${ }^{3}$ Huai'an Key Laboratory of Esophageal Cancer Biobank, ${ }^{4}$ Department of Gastroenterology, ${ }^{5}$ Department of Central Laboratory, ${ }^{6}$ Department of Gastrointestinal Surgery,

Huai'an First People's Hospital, Nanjing Medical University, Huai'an, ${ }^{7}$ Liver Transplantation Center, The First Affiliated Hospital of Nanjing Medical University, Nanjing, China

*These authors contributed equally to this work

Correspondence: Qi-long Wang Department of Clinical Oncology, Huai'an Key Laboratory of Esophageal

Cancer Biobank and Department of Central Laboratory, Huai'an First People's Hospital,

Nanjing Medical University, 6 W Beijing

Road, Huai'an 223300, China

Tel +86 I58 6I7I 0352

Email qlwang@njmu.edu.cn

Xiaofei Chen

Department of Clinical Oncology,

Huai'an First People's Hospital, Nanjing

Medical University, 6 W Beijing Road,

Huai'an 223300, China

Tel +86 I39 I5I0 700 I

Email cxf003835@I26.com

Xiao-jie Lu

Liver Transplantation Center, The First Affiliated Hospital of Nanjing Medical

University, 300 Guangzhou Road,

Nanjing 210029, China

$\mathrm{Tel}+86 I 7765 I 57178$

Email189@whu.edu.cn
This article was published in the following Dove Press journal:

International Journal of Nanomedicine

II September 2017

Number of times this article has been viewed

Abstract: Low toxicity and high efficacy are the key factors influencing the real-world clinical applications of nanomaterial-assisted drug delivery. In this study, novel hollow carbon spheres (HCSs) with narrow size distribution were developed. In addition to demonstrating their ease of synthesis for large-scale production, we also demonstrated in vitro that the HCSs possessed high drug-loading capacity, lower cell toxicity, and optimal drug release profile at low $\mathrm{pH}$, similar to the $\mathrm{pH}$ in the tumor microenvironment. The HCSs also displayed excellent immunocompatibility and could rapidly distribute themselves in the cytoplasm to escape lysosomal clearance. More importantly, the HCSs could efficiently deliver doxorubicin (a representative chemotherapeutic drug) to tumor sites, which resulted in significant inhibition of tumor growth in an esophageal xenograft cancer model. This also prolonged the circulation time and altered the biodistribution of the drug. In conclusion, this study revealed a novel drug delivery system for targeted tumor therapy.

Keywords: hollow carbon spheres, drug delivery, doxorubicin, esophagus carcinoma

\section{Introduction}

Nanovector-mediated drug delivery is of immense importance, since it can increase the therapeutic efficacy and reduce the side effects of the drug. ${ }^{1,2}$ With advancements in nanotechnology, several types of nanoparticles have been exploited for potential use in cancer therapy and diagnosis. Inorganic nanoparticles including gold nanoparticles, ${ }^{3,4}$ silver nanomaterials, ${ }^{5,6}$ silica nanocarriers, ${ }^{7,8}$ or iron oxide nanoparticles ${ }^{9}$ have been widely investigated for potential clinical applications. The growing interest in the use of inorganic nanomaterials in medicine is owing to their size- and shape-controllable preparation. However, the potential biohazards of engineered inorganic nanoparticles, including their impact on health and environment, have extensively limited their clinical applications. Therefore, researchers have shifted their focus from inorganic to organic nanomaterials, such as poly(lactic-co-glycolic acid), ${ }^{10,11}$ chitosan, ${ }^{12}$ polypeptides, ${ }^{13}$ liposomes, ${ }^{14}$ silk fibroin, ${ }^{15}$ lipids, ${ }^{16-18}$ and carbon-based nanomaterials. ${ }^{19,20}$

Recently, carbon nanomaterials including carbon nanotubes, ${ }^{21}$ carbon nanohorns (CNHs) ${ }^{22}$ carbon nanodiamonds, ${ }^{23}$ and graphene $(\mathrm{GN})^{24}$ have been studied for applications in drug delivery, owing to their excellent biocompatibility, superhydrophilicity, cell internalization property, enhanced permeability and retention (EPR) effect, and flexibility toward surface modifications. Yao et al developed a highly specific gastric cancer stem cell (CSC)-targeting drug delivery system based on single-wall carbon nanotubes (SWNTs), which could selectively and effectively eliminate gastric CSCs. ${ }^{25}$ 
Pérez-Martínez et al reported that the docetaxel-mediated cytotoxicity in human prostate cancer cells dramatically increased by conjugating the cofilin- 1 siRNA with CNHs. ${ }^{26}$ Cao et al developed an innovative carrier for miRNA-203 delivery in esophageal cancer cells using protamine sulfate (PS)-nanodiamond (ND) nanoparticles. The efficient delivery of $m i R-203$ by nanodiamonds significantly suppressed the proliferation and migration of cancer cells. ${ }^{27} \mathrm{Xu}$ et al proposed a novel drug delivery system for cancer therapy based on graphene oxide (GO) that displayed considerably higher cytotoxicity toward A549 and MCF-7 cells, over a broad range of paclitaxel (PTX) concentration and time, compared to free PTX.$^{28}$ Nevertheless, most carbon nanomaterials were solid in nature and their drug-loading capacities needed to be further improved.

In this study, we developed novel hollow carbon spheres (HCSs) using glucose. These spheres could be easily internalized into the cancer cells; however, this process could be inhibited by using phagocytosis- and autophagy-associated inhibitors. Environmental pH had no apparent effect on the uptake of HCSs by cancer cells. These spheres were nontoxic and displayed high drug-loading capacity and efficient delivery of doxorubicin (Dox, a representative therapeutic drug). The pharmacokinetic properties, release kinetics, and in vivo biodistribution data suggested that HCSs are excellent candidates for the delivery of cancer drugs.

\section{Materials and methods}

\section{Reagents}

Zinc nitrate hexahydrate, tetraethyl orthosilicate (TEOS), 2-methylimidazole, and Dox (a chemotherapeutic drug) were purchased from Sigma-Aldrich Co. (St Louis, MO, USA). Cyanine 5.5 carboxylic acid was procured from Lumiprobe (Hunt Valley, MD, USA). Isopropyl alcohol, ethanol, and sodium hydroxide were purchased from SINOPHARM (Beijing, China). RPMI 1640, fetal bovine serum, penicillin, and streptomycin for culturing cells were purchased from Thermo Fisher Scientific (Waltham, MA, USA).

\section{Mice}

Balb/c and SCID mice, 6-8 weeks of age, were purchased from the Experimental Animal Center of Nanjing Medical University. The animal experiments were conducted in accordance with the National Institutes of Health Guide for the Care and Use of Laboratory Animals.

\section{Cell culture}

The Eca109 cells were obtained from the Type Culture Collection of the Chinese Academy of Sciences, Shanghai,
China. The cells were maintained in RPMI 1640 medium supplemented with heat-inactivated FBS (10\%), penicillin $(50 \mathrm{IU} / \mathrm{mL})$, and streptomycin $(50 \mathrm{ng} / \mathrm{mL})$ and cultured in a humidified $\mathrm{CO}_{2}$ incubator at $37^{\circ} \mathrm{C}$.

\section{Synthesis of $\mathrm{SiO}_{2}$ spheres}

$\mathrm{SiO}_{2}$ spheres were synthesized using a slightly modified Stöber process. Briefly, TEOS $(0.6 \mathrm{~mL})$ was added to a mixture of isopropyl alcohol $(63.3 \mathrm{~mL})$, deionized water $(23.5 \mathrm{~mL})$, and aqueous ammonia $(30 \%, 13 \mathrm{~mL})$, and vigorously stirred at room $25^{\circ} \mathrm{C}$ for $1 \mathrm{~h}$. Next, TEOS $(5 \mathrm{~mL})$ was added dropwise into the solution at $35^{\circ} \mathrm{C}$ for $2 \mathrm{~h}$. Subsequently, the $\mathrm{SiO}_{2}$ spheres were collected from this suspension by centrifugation, washed several times with ethanol and deionized water, and dried in a vacuum oven (Thermo Fisher Scientific) at $70^{\circ} \mathrm{C}$ overnight.

\section{Synthesis of $\mathrm{SiO}_{2} @$ carbon core-shell spheres}

Briefly, $\mathrm{SiO}_{2}$ spheres (30 mg) were dispersed in deionized water $(30 \mathrm{~mL})$ by ultrasonication for $30 \mathrm{~min}$, followed by the addition of glucose $(0.5 \mathrm{~g})$. After stirring for $10 \mathrm{~min}$, the mixture was transferred to a $50-\mathrm{mL}$ Teflon-lined stainless steel autoclave and subsequently placed in an electric oven at $180^{\circ} \mathrm{C}$ for $12 \mathrm{~h}$. After cooling to room temperature, the product was washed thrice with ethanol, collected using centrifugation, and dried under vacuum at $80^{\circ} \mathrm{C}$.

\section{Synthesis of HCSs}

The $\mathrm{SiO}_{2} @$ carbon core-shell spheres thus prepared were soaked and washed in an aqueous solution of $\mathrm{NaOH}(3 \mathrm{M})$ at $70^{\circ} \mathrm{C}$ for $48 \mathrm{~h}$, following which the HCSs were obtained.

\section{Structural characterization}

The properties of HCSs were analyzed using the LabRAM HR 800 Raman spectrometer (HORIBA Scientific, Japan) and characterized using the Nicolet 6700 FT-IR spectrometer (Thermo Electron Corporation, PA, USA). The surface morphology and structure of the samples were observed using the Nova NanoSEM 230 scanning electron microscopy (SEM; FEI, OR, USA) and the JEM-2100F transmission electron microscopy (TEM; JEOL USA Inc., MA, USA).

\section{Dynamic light scattering (DLS)}

The HCSs were mixed with absolute ethanol and dispersed by sonicating in a bath sonicator (Shinva Medical Instrument Co., Ltd., China). Next, the size and zeta potential of the spheres were analyzed using the Nicomp ZLS Z3000 (FL, USA). 
Analysis of uptake of carbon spheres (CSs)

Cellular uptake of the CSs was examined using flow cytometry. The Eca109 cells were seeded in 6-well culture plates and cultured for $24 \mathrm{~h}$. The presonicated Cy5.5-labeled HCSs were added and incubated at $37^{\circ} \mathrm{C}$ for $12 \mathrm{~h}$ in dark. Next, the cells were collected after washing them three times with PBS, and cell fluorescence was analyzed using the FACSCalibur flow cytometer (BD Biosciences, San Jose, CA, USA).

To study the possible mechanisms of endocytosis, the cells were cultured at $37^{\circ} \mathrm{C}$ in the presence of an endocytosis inhibitor for $1 \mathrm{~h}$, followed by the addition of Cy5.5-HCSs. Next, the cells were cultured for $6 \mathrm{~h}$. The cultured cells were washed three times with PBS. The percentage of Cy5.5positive $\left(\mathrm{Cy} 5.5^{+}\right)$cells was quantified using fluorescenceactivated cell sorting (FACS).

To evaluate the cellular internalization and localization of HCSs, the Eca109 cells were plated onto the chamber slides (density, $5 \times 10^{4}$ cells/well) and cultured for $24 \mathrm{~h}$. Next, the Cy5.5-labeled HCS particles $(2 \mu \mathrm{g} / \mu \mathrm{L})$ were added and the culture was incubated for $6 \mathrm{~h}$. The cells were allowed to stain in the LysoTracker ${ }^{\circledR}$ Deep Red $(50 \mathrm{nM}$; Thermo Fisher Scientific) for $2 \mathrm{~h}$ at $37^{\circ} \mathrm{C}$. After incubation, the cells were washed three times with PBS (1×) and stained with DAPI. Next, the cells were fixed with PFA (4\%) for $10 \mathrm{~min}$ at RT, and the slides were imaged using the Nikon fluorescence microscope (NY, USA).

\section{In vitro and in vivo cytotoxicity assay}

For testing the in vitro toxicity of HCSs, the Eca109 cells were seeded onto the E-plates (density, 10,000 cells/well) in the culture medium and incubated at $37^{\circ} \mathrm{C}$ in $5 \% \mathrm{CO}_{2}$ overnight. Next, the HCS particles (final concentration: $5,10,20 \mu \mathrm{g} / \mathrm{mL}$ ) were added and the cell proliferation curves were automatically recorded in real-time using the xCELLigence System (Roche Applied Sciences, CA, USA). The cell index was followed for $40 \mathrm{~h}$.

For evaluating the in vivo toxicity of HCSs, Balb/c mice were injected intravenously (i.v.) with $\operatorname{HCSs}(5,10$, $20 \mathrm{mg} / \mathrm{kg}$ ). At 24-h post-injection, blood ( $0.5 \mathrm{~mL} / \mathrm{mouse})$ was collected for performing the blood chemistry tests, including white blood cell, red blood cell, and platelet counts. The body weight of each mouse was monitored after every 5 days for over 30 days.

\section{Drug loading and release analysis}

Briefly, HCSs (10 mg) were dispersed in the Dox aqueous solution ( $4 \mathrm{~mL}, 1 \mathrm{mg} / \mathrm{mL}$ ). The Dox-loaded sample was constantly stirred for $24 \mathrm{~h}$ in dark and collected by centrifugation.
Drug-loading efficiency was calculated according to the following equation.

Loading efficacy $(\%)=\frac{(\text { Total Dox }- \text { Unbound Dox })}{\text { Total Dox }} * 100$

The amount of drug released was studied by resuspending the HCS-Dox sample in PBS (3 mL, pH 7.4 or 5.5) at $37^{\circ} \mathrm{C}$ with gentle shaking. At each time point, PBS was removed and replaced with an equal volume of fresh PBS. The amount of Dox released in the supernatant was quantified using a UV-vis spectrophotometer (Thermo Fisher Scientific) at $497 \mathrm{~nm}$.

\section{Biodistribution and circulation time}

Mice were subcutaneously inoculated with the Eca109 cells $\left(5 \times 10^{6}\right.$ cells/mouse). The mice that developed tumors were i.v. administrated with Cy5.5-labeled HCS-Dox (Dox, $100 \mu \mathrm{g}$ ). For observing the accumulation of HCSs in the tumor, at 6-h post-injection, the Cy5.5-HCS-Dox administrated mice were scanned using the In-Vivo FX Pro image station (Bruker, NY, USA) and quantified using the Multiplex software (Bruker). To verify the circulation time, blood from the Cy5.5-HCS-Dox injected mice was collected into a tube (precoated with an anticoagulant) at various time points (1, 3, $6,12,24,48,72,96$, and $120 \mathrm{~h}$ ). The intensity of Cy 5.5 signal was measured using equal volumes of the blood samples by the In-Vivo FX Pro image station.

To analyze the Dox concentration in serum, mice were i.v. injected with either free Dox $(100 \mu \mathrm{g})$ or HCS-Dox (Dox, $100 \mu \mathrm{g}$ ). Serum from the peripheral blood samples was collected after $3,6,12,24,48,72,96,120$, and $144 \mathrm{~h}$. For analyzing Dox distribution in different tissues, the Dox $(100 \mu \mathrm{g})$ or HCS-Dox (Dox, $100 \mu \mathrm{g})$ injected mice were sacrificed after $24 \mathrm{~h}$. The organs, including liver, spleen, heart, lung, kidney, brain, and intestine, were homogenized. The concentration of Dox in serum and organs was measured using the Agilent 1100 HPLC system (Agilent, CA, USA).

\section{Tumor cell xenograft in SCID mice}

The experimental protocol was approved by the Institutional Animal Care and Use Committee of the Nanjing Medical University. The Eca109 cells $\left(5 \times 10^{6}\right)$ were suspended in ice-cold PBS $(50 \mu \mathrm{L})$ and subcutaneously inoculated into the left scapular region of 6-week-old SCID mice. When the tumor volume reached $\sim 100 \mathrm{~mm}^{3}$, the mice were randomly assigned to different treatment groups and i.v. injected with PBS, free HCSs $(20 \mathrm{mg} / \mathrm{kg})$, free Dox $(100 \mu \mathrm{g})$, or HCSs loaded with Dox (HCS-Dox; Dox, $100 \mu \mathrm{g}$ ). The mice were 
treated, as described above, after every 5 days for 10 times. Mortality was monitored daily and tumor growth was measured, spaced at 3-day intervals, using calipers. Tumor volume was calculated as follows:

$$
\text { Tumor volume }\left(\mathrm{mm}^{3}\right)=\frac{\left(\text { Length } \times \mathrm{Width}^{2}\right)}{2}
$$

\section{Statistical analysis}

The results were expressed as mean \pm standard deviation. One-way analysis of variance (ANOVA) followed by Tukey's post hoc test was used to determine the statistically significant differences between groups. The $t$-test was used to determine the differences between two groups $(* p<0.05$, $* * p<0.01, * * * p<0.001)$. Differences were considered to be statistically significant at $p<0.05$.

\section{Results and discussion}

\section{Preparation and characterization of HCS}

The HCSs were prepared from glucose using the hydrothermal method. Figure 1A represents the process and shows the following three steps for HCS preparation: 1) synthesis of $\mathrm{SiO}_{2}$ spheres, 2) synthesis of $\mathrm{SiO}_{2} @$ carbon core-shell spheres, and 3) synthesis of HCSs. Next, the morphology of HCSs was analyzed using SEM (Figure 1B) and TEM (Figure 1C). All the CSs exhibited similar spherical morphologies and uniform sizes. It was observed that the average diameter of the HCSs was $\sim 200 \mathrm{~nm}$.

The size and surface charge distribution of CSs was investigated using DLS. The average size (Figure 2A) and average zeta potential (Figure 2B) of the HCSs was observed to be $184 \mathrm{~nm}$ and $-31.72 \mathrm{mV}$, respectively. The average diameter calculated using DLS further supported the electron microscopy results. Zeta potential is one of the most important physical properties that is exhibited by the nanoparticles present in suspension. The particles with high zeta potential are more electrically stabilized compared to the particles with low zeta potential. This observation indicated that the HCSs synthesized by us were relatively stable. ${ }^{29}$

We used Raman and FTIR spectroscopy to identify the functional groups present in the HCSs. The Raman spectrum for the CSs is shown in Figure 3A. Two strong peaks at $1,585 \mathrm{~cm}^{-1}$ (the G-band) and $1,344 \mathrm{~cm}^{-1}$ (the D-band) were observed, which corresponded to the vibrations of crystalline graphite and disordered amorphous carbon, respectively. ${ }^{30,31}$ It is known that a higher IG/ID ratio facilitated effective loading of metal or therapeutic drugs. The FTIR spectrum shown in Figure 3B illustrates the functional groups present in HCSs after hydrothermal treatment. The bands at 1,708 and $1,625 \mathrm{~cm}^{-1}$ corresponded to $\mathrm{C}=\mathrm{O}$ and $\mathrm{C}=\mathrm{C}$ vibrations, respectively. ${ }^{32}$ This confirmed the existence of carbon, the hydrothermal carbonization of glucose, and also suggested the existence of large numbers of hydroxyl groups. Bands in the range of 1,000-1,400 $\mathrm{cm}^{-1}$ included the bending vibrations of $\mathrm{C}-\mathrm{OH}$ and $\mathrm{OH}$. Meanwhile, bands at $3,430 \mathrm{~cm}^{-1}$ were also attributed to the $\mathrm{OH}$ groups. The reductive functional groups, namely, $-\mathrm{OH}$ and - $\mathrm{CHO}$ groups, were bonded on the surface of carbon, which could improve the hydrophilicity and stability of the HCSs in aqueous systems. These data suggested that the HCSs could be functionalized with

A

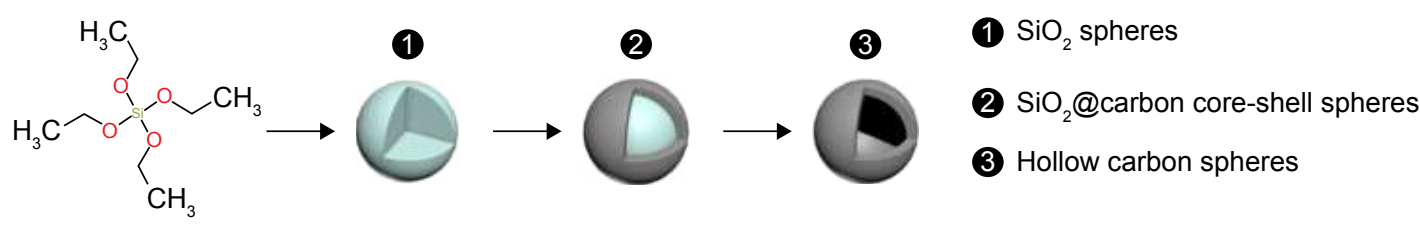

B

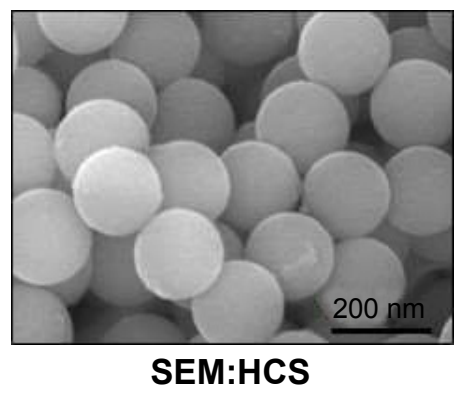

C

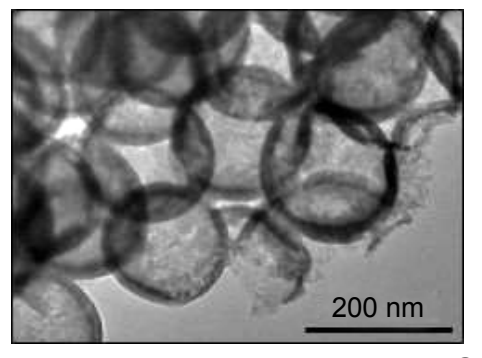

TEM:HCS

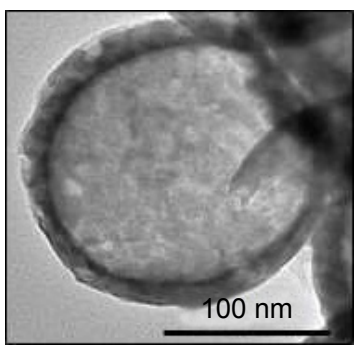

(B) SEM image of HCS (insert scale bar, $200 \mathrm{~nm}$ ). (C) TEM Figure I Preparation and analysis of morphology of HCSs. (A) Sch Abbreviations: HCS, hollow carbon sphere; SEM, scanning electron microscopy; TEM, transmission electron microscopy. 


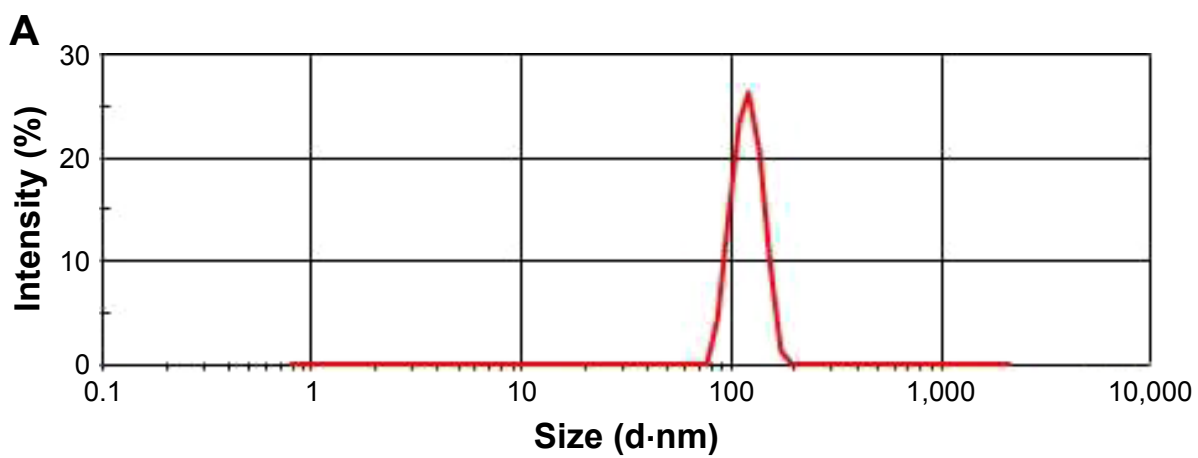

B

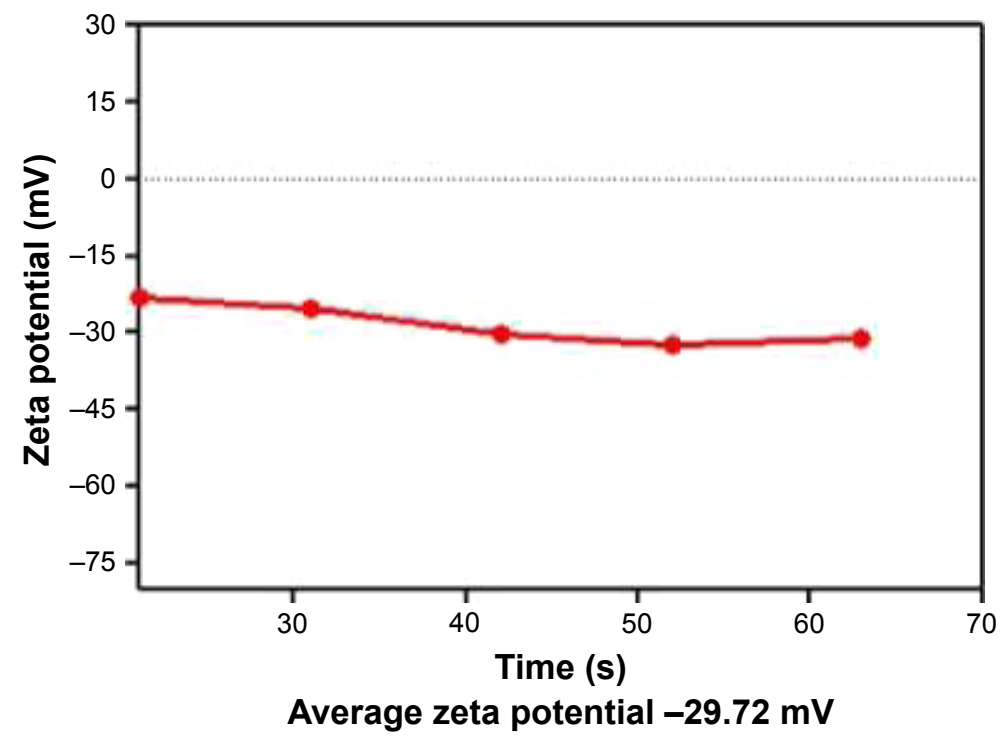

Figure 2 Characterization of HCSs. Analysis of $(\mathbf{A})$ size distribution of HCSs and $(\mathbf{B})$ surface zeta potential. Abbreviation: HCS, hollow carbon sphere.

hydroxyl, carbonyl, and carboxylic groups derived from the organic molecules. Thus, they were amenable to surface modifications, such as conjugation of chemotherapeutic drugs and targeting agents. ${ }^{33}$

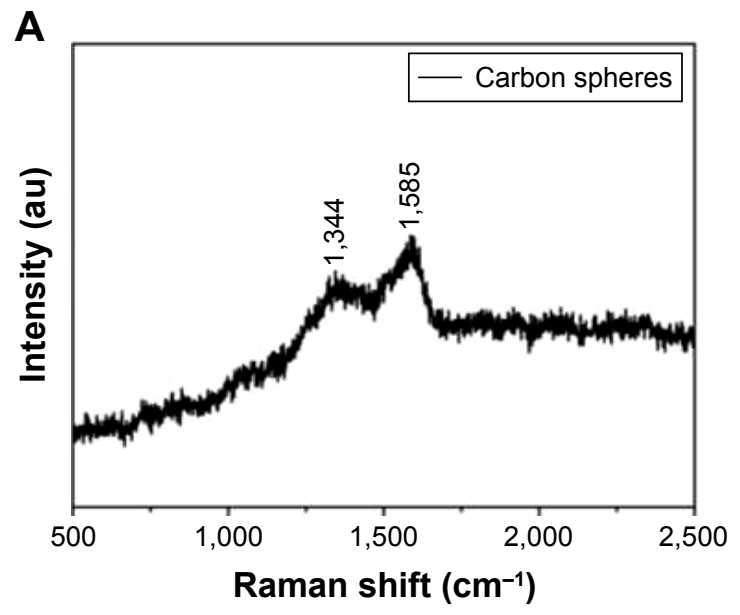

\section{Internalization and localization of HCS}

In order to evaluate the potential use of HCSs as vectors for delivering therapeutic agents, the efficient uptake of HCSs by Eca109 cells was evaluated. The Eca109 cells

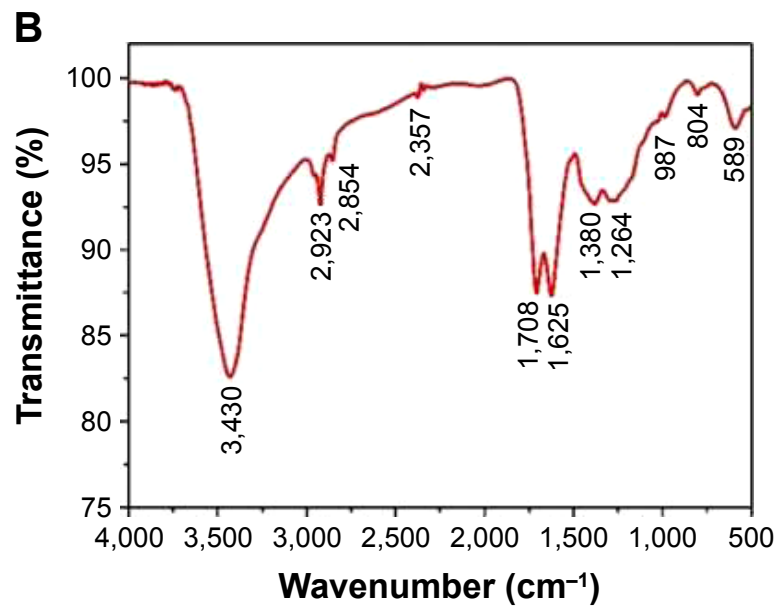

Figure 3 Structural characterization of HCSs. Properties of HCSs analyzed using (A) Raman spectroscopy and (B) FTIR spectroscopy. Abbreviation: HCS, hollow carbon sphere. 
were cocultured with Cy5.5-labeled HCSs. The presence of HCSs in cells was determined by quantitatively analyzing the Cy5.5- $\mathrm{HCS}^{+}$cells using FACS. As shown in Figure 4A, over $80 \%$ of the Eca109 cells were Cy5.5-HCS ${ }^{+}$; however, the uptake of Cy5.5-HCSs dramatically decreased upon treatment with bafilomycin A1 (a macrolide antibiotic that prevents the maturation of autophagic vacuoles) and cytochalasin D (an inhibitor of microfilament formation required for phagocytosis) (Figure 4B). Furthermore, as shown in Figure $4 \mathrm{C}$, pH did not considerably affect the uptake of HCSs by Eca109 cells.

Lysosomal degradation-mediated elimination of the unnecessary endogenous and exogenous materials by cells is a bottleneck for most nanomaterials. ${ }^{34,35}$ Escaping lysosomal degradation is a desirable characteristic for drug delivery applications. Thus, localization of the HCSs was assessed using a confocal laser scanning microscopy. The Cy5.5-HCSs were prepared, and the fluorescence signal was scanned using an in vivo imaging system (Figure 5A), for determining the localization of Cy5.5-HCSs in the Eca109 cells. As shown in Figure 5B, the Cy5.5-HCSs (green) were uniformly distributed in the cells. Additionally, their accumulation in the lysosomes (red) was not observed in the cells. This property makes HCSs more suitable for use in drug delivery.

\section{Ex vivo and in vivo toxicity assays}

Toxicity remains a key issue that limits the wide applicability of nanomaterials. ${ }^{36}$ To further explore their potential for clinical use, the in vitro experiments were performed to evaluate the effect of HCSs on cell proliferation. In the in vivo experiments, changes in body weight and blood chemistry of the HCS-administrated mice were observed. As shown in Figure 6A, HCSs (at varying concentrations) showed no apparent effect on cell proliferation. The mean body weight (Figure 6B) and blood cells (Figure 6C) of the HCS-injected Balb/c mice were similar to those of the control mice (Balb/c mice without HCS administration). This result was in accordance with the results reported by Bayda et al. ${ }^{37}$

\section{Drug-loading capacity and release profile}

Other desirable properties of nanomaterials include high drug-loading capacity and controlled drug release under physiological conditions. In order to evaluate the therapeutic drug-loading efficacy, equal amounts of HCSs or control CSs were mixed with equal amounts of doxorubicin and stirred at
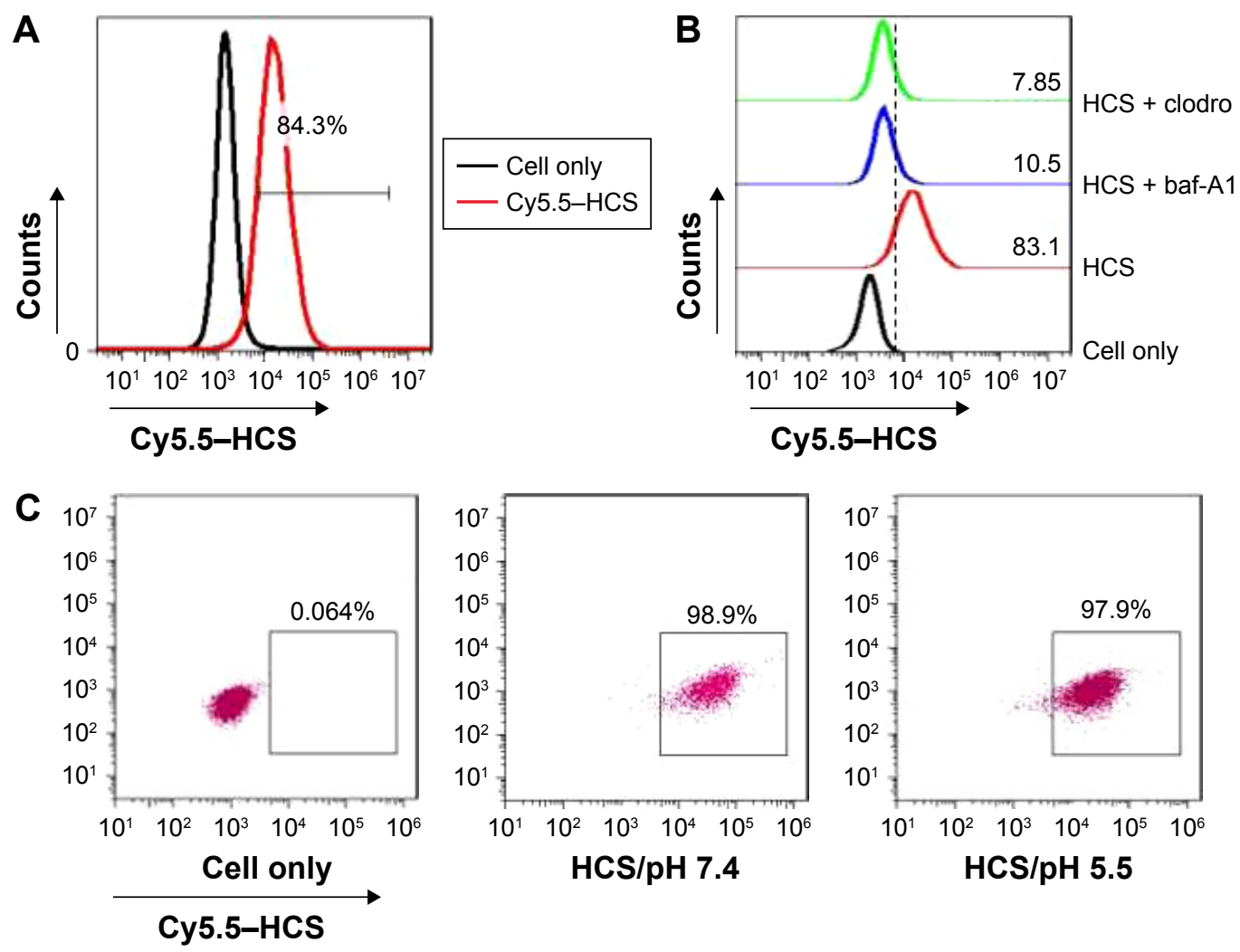

Figure 4 Internalization of HCSs. (A) Internalization of Cy5.5-HCSs in Ecal09 cells examined using FACS. (B) Analysis of the potential mechanism of Cy5.5-HCS endocytosis in Eca 109 cells. (C) Effect of pH on the uptake of HCSs by Eca 109 cells.

Abbreviations: HCS, hollow carbon sphere; FACS, fluorescence-activated cell sorting. 
A

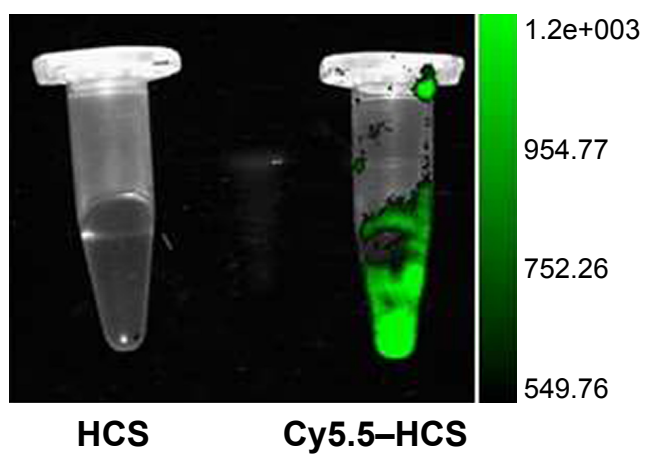

B

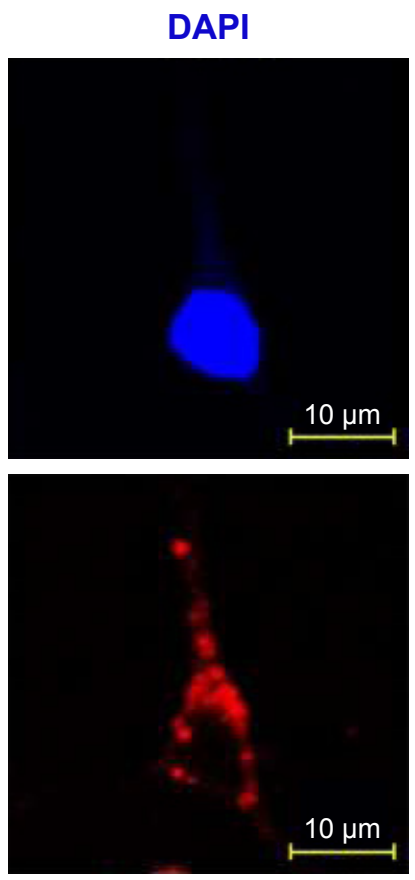

LysoTracker
Cy5.5-HCS
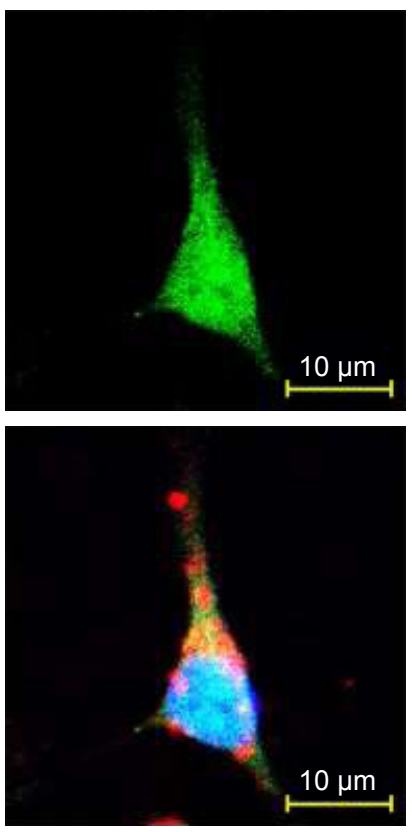

Merge

Figure $\mathbf{5}$ Localization of HCSs in cells. (A) Cy5.5-labeled HCSs were prepared and the fluorescent signals were measured using an in vivo imaging system. (B) Eca 09 cells treated with Cy5.5-HCSs (green) and marked with LysoTracker (red). Scale bar $=10 \mu \mathrm{m}$.

Abbreviation: HCS, hollow carbon sphere.
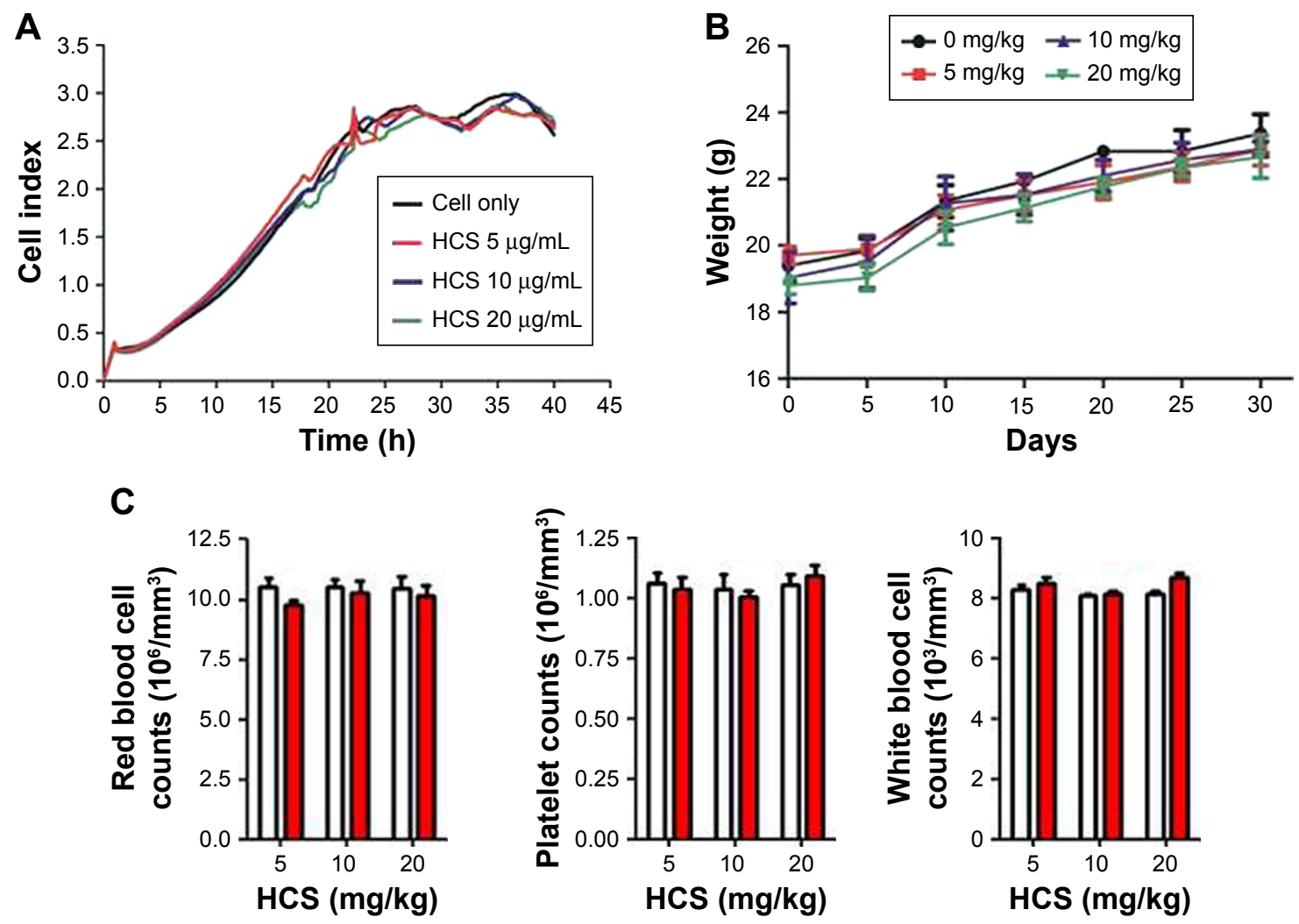

Figure 6 Toxicity of HCSs. (A) In vitro cell proliferation assay to evaluate the cytotoxicity of HCSs. In vivo toxicity assay for analyzing (B) body weight and (C) blood cells chemistry in mice.

Abbreviation: HCS, hollow carbon sphere. 
room temperature. The free drug was removed and the drugloaded spheres were photographed (Figure 7A). The loading efficacy of HCSs was calculated to be $\sim 74 \%$ compared to $56 \%$ for CSs (Figure $7 \mathrm{~B} ;{ }^{* *} p<0.01$ ). This indicated the significantly higher drug-loading capacity of HCSs compared to CSs (in the present study) or to the carbon nanoparticles developed by the other groups.

Previous studies have reported that the extracellular $\mathrm{pH}$ of tumor tissues was significantly lower ( $\mathrm{pH}$ 6-6.9) than that of normal tissues ( $\mathrm{pH}$ 7.3-7.4). The key factor responsible for this phenomenon was the specialized metabolism in tumor cells. ${ }^{38,39}$ Most tumor cells metabolized glucose or other substrates to lactic acid. As a result, they pumped lactic acid and protons into the extracellular space in order to regulate the intracellular $\mathrm{pH}$. Thus, numerous $\mathrm{pH}$-responsive nanomaterials were developed..$^{40,41}$ To study the release of Dox by HCS-Dox, the release experiment was performed in the $\mathrm{pH}$ range of 5.5-7.4. The results revealed that Dox release was comparatively slower at alkaline $\mathrm{pH}(\mathrm{pH}$ 7.4) as shown in Figure $7 \mathrm{C}(* p<0.05 ; * * p<0.01)$. This observation was consistent with the observations reported by Bayda et $\mathrm{al}^{37}$ and Qiu et al. ${ }^{42}$ Dox is a weak amphipathic base. The protonated form of Dox is $\sim 10$-fold that of free base. Hence, the interaction between Dox and carbon nanoparticles is considerably strong under physiological conditions. In an acidic environment, the carboxylic groups present on the particle surface get dissociated. As a result, the negative charge on the surface decreases and the interaction is weakened. Finally, this results in increased drug release.

\section{Biodistribution of HCSs in vivo}

Pharmacokinetics and biodistribution of drugs can be changed by using nanotechnology. Chemotherapeutic drugs do not specifically target the cancerous cells. Thus, to achieve target specificity and improve the efficacy of chemotherapeutic drugs, nanovectors remain the best choice. Efficient delivery of drugs to tumor sites relies heavily on the EPR effect of nanocarriers. To understand the potential redistribution of Dox delivered by HCSs, in vivo fluorescence imaging was performed. The circulation time and distribution of drug in organs and tumor tissues were analyzed. As shown in Figure 8A, the whole mouse body images clearly indicated that HCS-Dox accumulated in the tumor tissue, $6 \mathrm{~h}$ after the administration of the drug in tail vein. The circulation time was also measured by scanning peripheral blood of the mice injected with HCS-Dox. As shown in Figure 8B, HCS-Dox could be detected up to 5 days. The elimination of Dox from blood was significantly prolonged upon administration as HCS-Dox compared to that with free Dox (Figure $8 \mathrm{C}$; ${ }^{*} p<0.05$ ). Tissue distribution of Dox (Figure 8D) demonstrated that HCSs increased the accumulation of Dox in the liver and tumor tissues and reduced its accumulation in the heart and kidney tissues $(* p<0.05$ and $* * p<0.01)$. These data suggested that HCS-Dox could reduce Dox toxicity toward the heart and kidney tissues, along with increasing its antitumor activities.

\section{In vivo anticancer activity}

Esophageal cancer presents one of the most aggressive malignancies. Despite advances in surgical techniques and radiation therapy, the 5-year survival rate of patients with esophageal cancer remains poor. Over the past decade, researchers have focused their efforts on targeted drug development; however, the number of approved targeted drugs for esophageal cancer remains few. ${ }^{43}$ In this study, the antitumor property of HCS-Dox was evaluated in SCID mice bearing Eca109 tumors. As shown in Figure 9A, the randomly grouped tumor-bearing mice were treated with
A

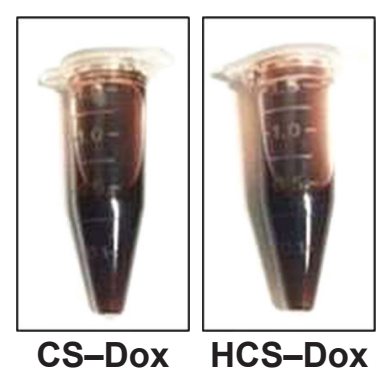

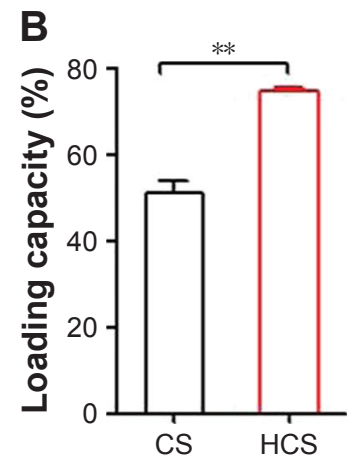

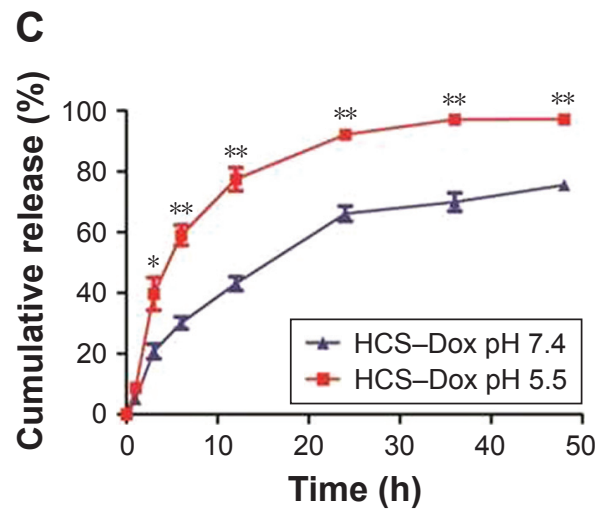

Figure 7 Drug-loading capacity and drug-releasing profile of HCSs. (A) Solid CSs and HCSs loaded with Dox were prepared and photographed. (B) Calculation of the loading percentage of Dox and the difference in loading capacity between CSs and HCSs. HCSs displayed more efficient drug-loading capacity. (C) Measurement of cumulative release of Dox from HCS-Dox evaluated at $\mathrm{pH} 5.5-7.4$. $* p<0.05$, ** $p<0.01$.

Abbreviations: HCS, hollow carbon sphere; CS, carbon sphere; Dox, doxorubicin. 
A
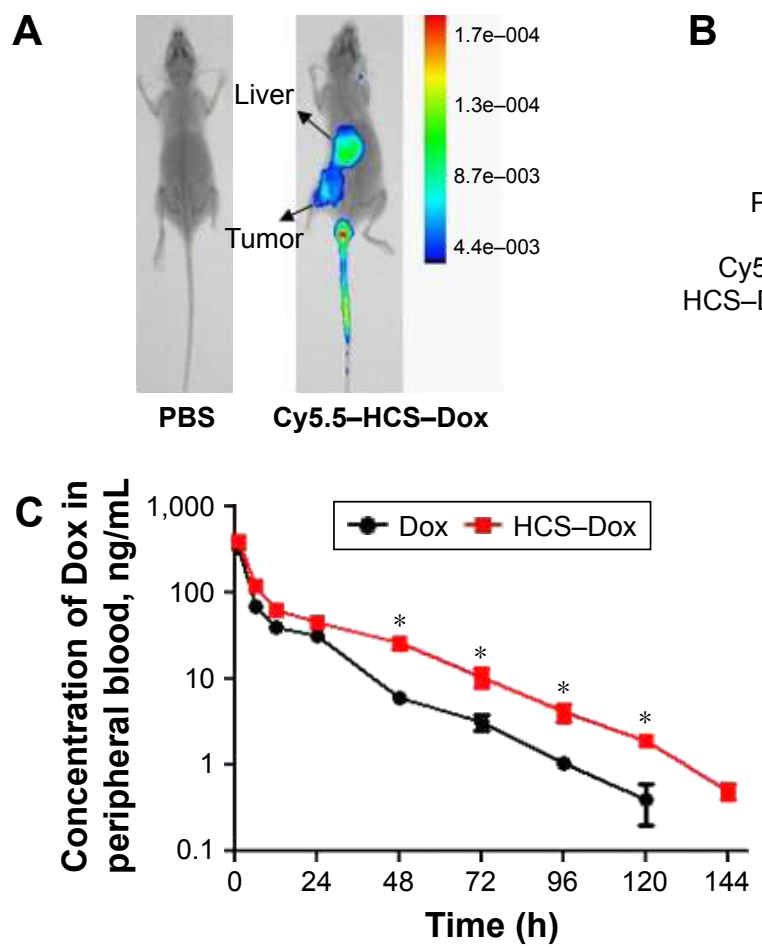
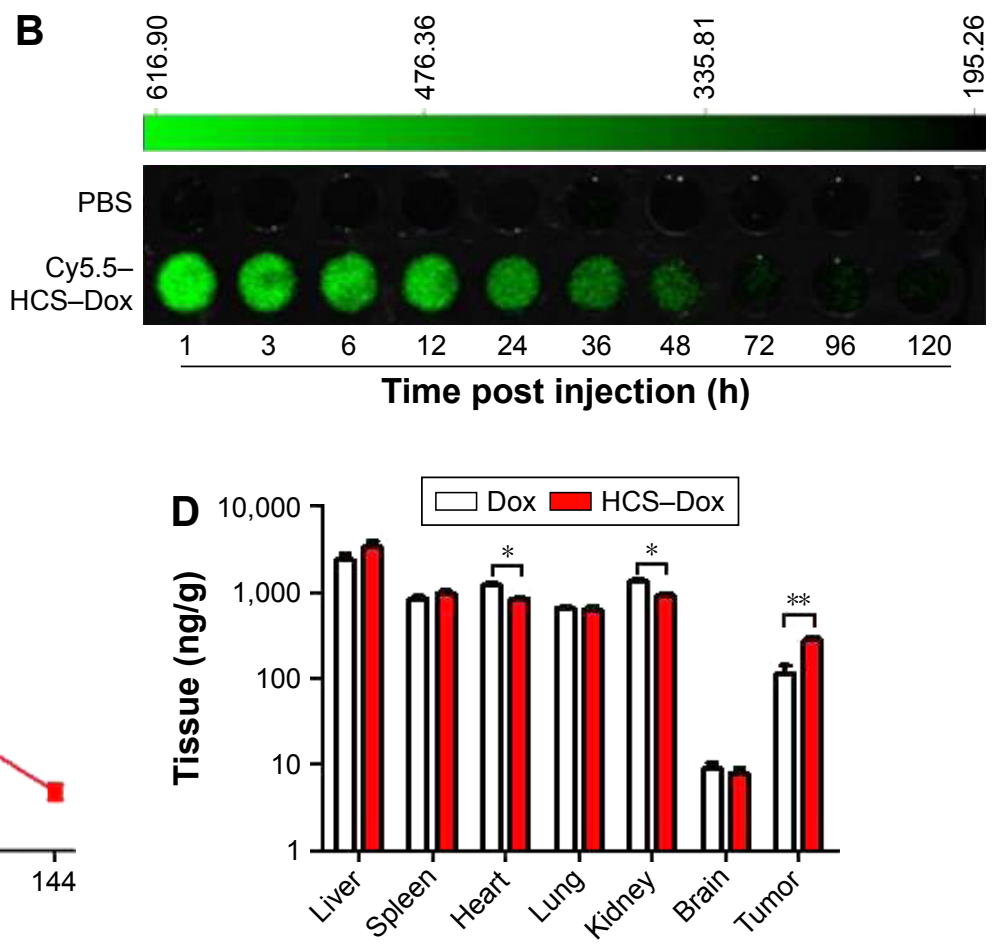

Figure 8 Biodistribution and circulation time of HCSs. (A) Cy5.5-labeled HCSs were prepared and i.v. injected into the Eca 109 tumor-bearing mice, and the whole mouse body was scanned using an in vivo imaging system. The (B) circulation time and (C) quantity of Dox in peripheral blood were analyzed at different time points after HCS-Dox administration. (D) Distribution of Dox in different organs of the mice including liver, spleen, heart, lung, kidney, brain, and tumor tissues. ${ }^{*} p<0.05$; $* * p<0.0$ I. Abbreviations: HCS, hollow carbon sphere; i.v., intravenously; Dox, doxorubicin.

A
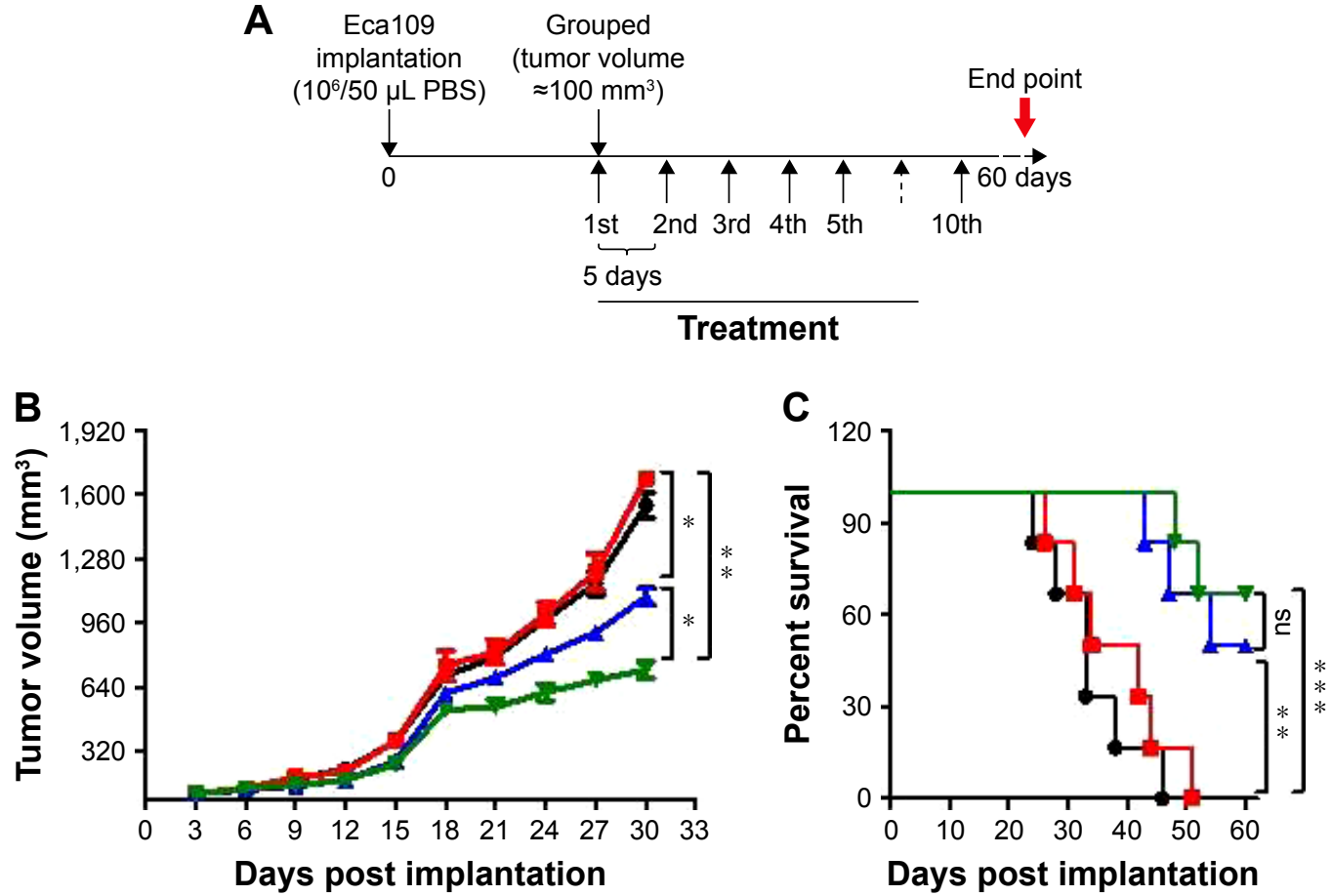

Figure 9 In vivo antitumor activity. An esophageal cancer xenograft model was established to verify the application of HCSs in cancer therapy. (A) Protocol for tumor implantation and treatment. Measurement of $(\mathbf{B})$ tumor volume and $(\mathbf{C})$ survival at 3 -day intervals in tumor-bearing mice, monitored for 60 days. $* p<0.05$; $* * p<0.0$ I; $* * * p<0.001$.

Abbreviations: HCS, hollow carbon sphere; Dox, doxorubicin; ns, not significant. 
PBS, free HCSs, free Dox, or HCS-Dox at an identical dose of $100 \mu \mathrm{g}$ Dox for 10 times. Dox bound to HCSs exerted a more effective antitumor effect (evident by tumor growth suppression) compared to free Dox (Figure 9B, ${ }^{*} p<0.05$ and $* * p<0.01)$. The lifespan of HCS-Dox-treated mice was significantly prolonged compared to PBS and HCS groups (Figure $9 \mathrm{C},{ }^{* *} p<0.01$ and $* * * p<0.001$ ), thereby indicating that HCSs can serve as a promising vector for the delivery of chemotherapeutic drugs.

\section{Conclusion}

In summary, this work reports novel HCSs developed using glucose. These spheres exhibited higher drug-loading capacity compared to the solid CSs. Other desirable properties of HCSs included the ability for escaping lysosomal degradation and controlled therapeutic drug release under acidic conditions. Most importantly, HCS-Dox could significantly promote tumor suppression owing to its EPR effect and longer circulation time. These results suggested that the HCSs could serve as a promising drug carrier for cancer therapy.

\section{Acknowledgments}

We would like to thank Dr Weiyong Yu and Miss Jiru Wang for providing assistance with this study. This work was supported by the National Natural Science Foundation of China (grant numbers 81572977, 81772585, and 81572421), Natural Science Foundation of Jiangsu Province (grant number BK20150421), International S\&T Cooperation Projects of Huai'an (grant number HAC2015015), and Huai'an Key Laboratory of Esophageal Cancer Biobank Project (grant number HAP201606).

\section{Disclosure}

The authors report no conflicts of interest in this work.

\section{References}

1. Wilczewska AZ, Niemirowicz K, Markiewicz KH, Car H. Nanoparticles as drug delivery systems. Pharmacol Rep. 2012;64(5):1020-1037.

2. Ramasamy T, Ruttala HB, Gupta B, et al. Smart chemistry-based nanosized drug delivery systems for systemic applications: a comprehensive review. J Control Release. 2017;258:226-253.

3. Elbialy NS, Fathy MM, Khalil WM. Doxorubicin loaded magnetic gold nanoparticles for in vivo targeted drug delivery. Int J Pharm. 2015;490(1-2):190-199.

4. Park H, Tsutsumi H, Mihara H. Cell-selective intracellular drug delivery using doxorubicin and $\alpha$-helical peptides conjugated to gold nanoparticles. Biomaterials. 2014;35(10):3480-3487.

5. Ong C, Lim JZ, Ng CT, Li JJ, Yung LY, Bay BH. Silver nanoparticles in cancer: therapeutic efficacy and toxicity. Curr Med Chem. 2013; 20(6):772-781.
6. Nair GG, Nair CK. Sanazole directed targeting of silver nanoparticle drug complex to tumor mass: a preclinical investigation in murine model. J Cancer Res Ther. 2014;10(4):979-984.

7. Qu Q, Ma X, Zhao Y. Targeted delivery of doxorubicin to mitochondria using mesoporous silica nanoparticle nanocarriers. Nanoscale. 2015;7(40):16677-16686.

8. Tarn D, Ashley CE, Xue M, Carnes EC, Zink JI, Brinker CJ. Mesoporous silica nanoparticle nanocarriers: biofunctionality and biocompatibility. Acc Chem Res. 2013;46(3):792-801.

9. Laurent S, Saei AA, Behzadi S, Panahifar A, Mahmoudi M. Superparamagnetic iron oxide nanoparticles for delivery of therapeutic agents: opportunities and challenges. Expert Opin Drug Deliv. 2014;11(9): $1449-1470$.

10. Fornaguera C, Dols-Perez A, Calderó G, García-Celma MJ, Camarasa J, Solans C. PLGA nanoparticles prepared by nano-emulsion templating using low-energy methods as efficient nanocarriers for drug delivery across the blood-brain barrier. J Control Release. 2015;211:134-143.

11. Kapoor DN, Bhatia A, Kaur R, Sharma R, Kaur G, Dhawan S. PLGA: a unique polymer for drug delivery. Ther Deliv. 2015;6(1):41-58.

12. Prabaharan M. Chitosan-based nanoparticles for tumor-targeted drug delivery. Int J Biol Macromol. 2015;72:1313-1322.

13. Ramasamy T, Ruttala HB, Chitrapriya N, et al. Engineering of cell microenvironment-responsive polypeptide nanovehicle co-encapsulating a synergistic combination of small molecules for effective chemotherapy in solid tumors. Acta Biomater. 2017;48:131-143.

14. Allen TM, Cullis PR. Liposomal drug delivery systems: from concept to clinical applications. Adv Drug Deliv Rev. 2013;65(1):36-48.

15. Mottaghitalab F, Farokhi M, Shokrgozar MA, Atyabi F, Hosseinkhani H. Silk fibroin nanoparticle as a novel drug delivery system. J Control Release. 2015;206:161-176.

16. Wang Q, Ren Y, Mu J, et al. Grapefruit-derived nanovectors use an activated leukocyte trafficking pathway to deliver therapeutic agents to inflammatory tumor sites. Cancer Res. 2015;75(12):2520-2529.

17. Wang Q, Zhuang X, Mu J, et al. Delivery of therapeutic agents by nanoparticles made of grapefruit-derived lipids. Nat Commun. 2013; $4: 1867$.

18. Sundaramoorthy P, Ramasamy T, Mishra SK, et al. Engineering of caveolae-specific self-micellizing anticancer lipid nanoparticles to enhance the chemotherapeutic efficacy of oxaliplatin in colorectal cancer cells. Acta Biomater. 2016;42:220-231.

19. Lim DJ, Sim M, Oh L, Lim K, Park H. Carbon-based drug delivery carriers for cancer therapy. Arch Pharm Res. 2014;37(1):43-52.

20. Chakrabarti M, Kiseleva R, Vertegel A, Ray SK. Carbon nanomaterials for drug delivery and cancer therapy. J Nanosci Nanotechnol. 2015; 15(8):5501-5511.

21. Iannazzo D, Piperno A, Pistone A, Grassi G, Galvagno S. Recent advances in carbon nanotubes as delivery systems for anticancer drugs. Curr Med Chem. 2013;20(11):1333-1354.

22. Nakamura M, Tahara Y, Ikehara Y, et al. Single-walled carbon nanohorns as drug carriers: adsorption of prednisolone and anti-inflammatory effects on arthritis. Nanotechnology. 2011;22(46):465102.

23. Kaur R, Badea I. Nanodiamonds as novel nanomaterials for biomedical applications: drug delivery and imaging systems. Int J Nanomedicine. 2013;8:203-220.

24. Yang K, Feng L, Liu Z. The advancing uses of nano-graphene in drug delivery. Expert Opin Drug Deliv. 2015;12(4):601-612.

25. Yao HJ, Zhang YG, Sun L, Liu Y. The effect of hyaluronic acid functionalized carbon nanotubes loaded with salinomycin on gastric cancer stem cells. Biomaterials. 2014;35(33):9208-9223.

26. Pérez-Martínez FC, Carrión B, Lucío MI, et al. Enhanced docetaxelmediated cytotoxicity in human prostate cancer cells through knockdown of cofilin-1 by carbon nanohorn delivered siRNA. Biomaterials. 2012;33(32):8152-8159.

27. Cao M, Deng X, Su S, et al. Protamine sulfate-nanodiamond hybrid nanoparticles as a vetcor for MiR-203 restoration in esophageal carcinoma cells. Nanoscale. 2013;5(24):12120-12125. 
28. Xu Z, Zhu S, Wang M, Li Y, Shi P, Huang X. Delivery of paclitaxel using PEGylated graphene oxide as a nanocarrier. ACS Appl Mater Interfaces. 2015;7(2):1355-1363.

29. Zhang Y, Yang M, Portney NG, et al. Zeta potential: a surface electrical characteristic to probe the interaction of nanoparticles with normal and cancer human breast epithelial cells. Biomed Microdevices. 2008; 10(2):321-328.

30. Ferrari AC, Robertson J. Interpretation of Raman spectra of disordered and amorphous carbon. Phys Rev B. 2000;61:14095-14107.

31. Sevilla M, Fuertes, Antonio B. Chemical and structural properties of carbonaceous products obtained by hydrothermal carbonization of saccharides. Chem Eur J. 2009;15:4195-4203.

32. Sun X, Li Y. Colloidal carbon spheres and their core/shell structures with noble-metal nanoparticles. Angew Chem Int Ed. 2004;43: 597-601.

33. Lim DJ, Sim M, Oh L, Lim K, Park H. Carbon-based drug delivery carriers for cancer therapy. Arch Pharm Res. 2014;37(1):43-52.

34. Canton I, Battaglia G. Endocytosis at the nanoscale. Chem Soc Rev. 2012;41(7):2718-2739.

35. Fröhlich E. Cellular elimination of nanoparticles. Environ Toxicol Pharmacol. 2016;46:90-94.
36. Zoroddu MA, Medici S, Ledda A, Nurchi VM, Lachowicz JI, Peana M Toxicity of nanoparticles. Curr Med Chem. 2014;21(33):3837-3853.

37. Bayda S, Hadla M, Palazzolo S, et al. Bottom-up synthesis of carbon nanoparticles with higher doxorubicin efficacy. $J$ Control Release. 2017;248:144-152.

38. McIntyre A, Harris AL. The role of $\mathrm{pH}$ regulation in cancer progression. Recent Results Cancer Res. 2016;207:93-134.

39. Lee ES, Gao Z, Bae YH. Recent progress in tumor $\mathrm{pH}$ targeting nanotechnology. J Control Release. 2008;132(3):164-170.

40. Meng F, Zhong Y, Cheng R, Deng C, Zhong Z. pH-sensitive polymeric nanoparticles for tumor-targeting doxorubicin delivery: concept and recent advances. Nanomedicine (Lond). 2014;9(3):487-499.

41. Xu D, Wu F, Chen Y, Wei L, Yuan W. pH-sensitive degradable nanoparticles for highly efficient intracellular delivery of exogenous protein Int J Nanomedicine. 2013;8:3405-3414

42. Qiu J, Zhang R, Li J, et al. Fluorescent graphene quantum dots as traceable, $\mathrm{pH}$-sensitive drug delivery systems. Int $J$ Nanomedicine. 2015;10:6709-6724.

43. Mohamed A, El-Rayes B, Khuri FR, Saba NF. Targeted therapies in metastatic esophageal cancer: advances over the past decade. Crit Rev Oncol Hematol. 2014;91(2):186-196.
International Journal of Nanomedicine

\section{Publish your work in this journal}

The International Journal of Nanomedicine is an international, peerreviewed journal focusing on the application of nanotechnology in diagnostics, therapeutics, and drug delivery systems throughou the biomedical field. This journal is indexed on PubMed Central, MedLine, CAS, SciSearch $\AA$, Current Contents $₫ /$ Clinical Medicine,

\section{Dovepress}

Journal Citation Reports/Science Edition, EMBase, Scopus and the Elsevier Bibliographic databases. The manuscript management system is completely online and includes a very quick and fair peer-review system, which is all easy to use. Visit http://www.dovepress.com/ testimonials.php to read real quotes from published authors. 